\title{
Change in Behavior Problems from Childhood Through Adolescence for Children with Fragile X Syndrome
}

\author{
Leann Smith DaWalt ${ }^{1} \cdot$ Heather Fielding-Gebhardt ${ }^{2} \cdot$ Kandace K. Fleming $^{2} \cdot$ Steven F. Warren ${ }^{2} \cdot$ Nancy Brady $^{2}$
}

Accepted: 1 September 2021 / Published online: 18 September 2021

(c) The Author(s), under exclusive licence to Springer Science+Business Media, LLC, part of Springer Nature 2021

\begin{abstract}
In this study, we examined trajectories of specific domains of behavior problems (i.e., attention problems, depression/anxiety, and aggressive behavior) from age 6 to 18 in a sample of 55 children with fragile $\mathrm{X}$ syndrome. We also examined autism status and early parenting as predictors of subsequent behavioral trajectories. We found that attention problems and aggressive behavior declined steadily from childhood through adolescence whereas anxious/depressed behavior demonstrated relative stability over the same period. Youth with highly flexible mothers displayed more optional trajectories of improvement in attention problems.
\end{abstract}

Keywords Fragile X syndrome $\cdot$ Behavior problems $\cdot$ Autism $\cdot$ Parenting $\cdot$ Adolescence

$\begin{array}{ll}\text { Abbreviations } \\ \text { ASD } & \text { Autism spectrum disorder } \\ \text { CARS } & \text { Childhood Autism Rating Scale } \\ \text { CBCL } & \text { Child Behavior Checklist } \\ \text { FXS } & \text { Fragile X syndrome } \\ \text { ICC } & \text { Intraclass correlations } \\ \text { IDD } & \text { Intellectual and developmental disability }\end{array}$

\section{Introduction}

Fragile X syndrome (FXS) is the leading known inherited cause of intellectual disability, affecting as many as 1 in 4000 males and 1 in 8000 females (Crawford et al., 2001), with recent studies suggesting even higher rates (up to 1 in 2500; Fernandez-Carvajal et al., 2009; Hagerman, 2008). It is estimated that the prevalence for co-occurring autism spectrum disorder (ASD) for individuals with FXS is around 43-75\% for males and 16-45\% for females (Bailey et al., 2008; Demark et al., 2003; Harris et al., 2008; Hernandez et al., 2009). The behavioral phenotype of FXS is marked by a range of challenging behaviors and mental health

Leann Smith DaWalt

lesmith@wisc.edu

1 Waisman Center, University of Wisconsin-Madison, 1500 Highland Ave., Madison, WI 53705, USA

2 University of Kansas, Lawrence, KS, USA symptoms including aggression, hyperactivity and inattention, anxiety, and depression (Bailey et al., 2008; Hardiman \& McGill, 2018). However, little is known about how these behaviors may change across childhood, particularly as children enter into the adolescent years. The present study addressed this gap by longitudinally examining change in reported behavior problems for a cohort of children with FXS from ages 6 to 18 years of age.

Few studies have utilized longitudinal methods to examine behavioral profiles of children with FXS, particularly during the transitional years of adolescence. Also, little attention has been given to the specific behavioral challenges and their unique trajectories over time. The work that has been conducted suggests that behavior may improve with age, or at least does not appear to become more severe. For example, a longitudinal study of behavior problems in boys with FXS suggests that challenging behavior may remain stable over a three-year period during childhood (Hatton et al., 2002). In contrast, in a study of individuals with FXS ranging from 2 to 26 years of age over a 6 year period, Hustyi and colleagues found decreases in several behavior problem domains, including hyperactivity, inappropriate speech, and social withdrawal (Hustyi et al. 2014). Similarly, Usher et al. (2020) followed adolescents and adults with FXS over nearly a 10-year period of time and observed that behavior problems became less severe as individuals aged. However, in this study, the sample did not include children and examined a total behavior problem score, rather than specific behaviors. In our own work (Fielding-Gebhardt et al., 2020), 
we recently examined the impact of challenging child behaviors on maternal well-being over time. We examined changes in total scores from the Child Behavior Checklist (CBCL; Achenbach \& Rescorla, 2001) across three time points, starting when children were, on average, 9 years old until they were 16 years old. We found that challenging behaviors significantly decreased over time, however we did not examine changes in specific behavioral challenges. We also did not explore predictors of child behavior.

\section{Autism and the Behavioral Phenotype of FXS}

Previous literature has found co-occurring ASD to be a significant predictor of behavioral outcomes for individuals with FXS (Hartley et al., 2011; Kaufmann et al., 2004; McDuffie et al., 2010). For example, in a cross-sectional study by Smith and colleagues, adolescents and adult with FXS and ASD had higher levels of behavior problems than did those with ASD only or FXS only (Smith et al., 2012a, 2012b). There also is some evidence that higher levels of ASD symptoms may be associated with increases in problem behavior over time in individuals with FXS (Crawford et al. 2018), although the impact on trajectories of behavior is unclear. For example, in a sample of adolescents and adults with FXS, Usher et al. (2020) found autism symptoms to predict a greater severity of behavior problems but these symptoms did not predict change in behavior problems. More research is needed to understand patterns of change for key aspects of the behavioral phenotype in children with FXS and the impact of both autism and parenting on these trajectories.

\section{Parenting Behavior and FXS}

An additional predictor of presence of and change in behavior problems in FXS may be parenting. In addition to our work examining challenging behaviors in our sample of children and adolescents with FXS, we have explored the effects of parenting, specifically maternal responsivity, on child development (Brady et al., 2014; Warren et al., 2012; Warren et al., 2017). Maternal responsivity can be defined at multiple levels (Warren \& Brady, 2007) and can have a positive effect on many domains of development. To start, responsivity can be characterized at the molar level by qualities of warmth, affect, and flexibility (see Sterling et al., 2013; Warren \& Brady, 2007). At a narrower, molecular level, maternal responsivity includes specific behaviors such as contingent responses and following the child's lead. Child communication, both broadly (Warren et al., 2017) and specifically (i.e., receptive vocabulary and number of different words; Warren et al., 2010 and Brady et al., 2014), benefits from highly-responsive maternal behaviors. This effect endures even when controlling for symptoms of autism and nonverbal cognition. Similarly, maternal responsivity has a positive effect on child social and daily living skills in children with FXS (Warren et al., 2017). However, we have not yet explored the impact of early maternal parenting on challenging behaviors in FXS.

\section{Present Study}

Attention problems, anxiety, depression, and aggressive behavior are common challenges associated with FXS. However, little is known about how these behaviors may change across childhood and adolescence. Further, despite the strong associations between parenting and behavioral development in children with typical development, the impact of early parenting on behavioral trajectories in children with FXS is also poorly understood. In this study, we examined trajectories of specific domains of behavior problems (i.e., attention problems, depression/anxiety, and aggressive behavior) from age 6 to 18 in a sample of children with FXS. We also considered autism status and early parenting as predictors of subsequent behavioral trajectories. We hypothesized that behavioral challenges would decline by the end of the study period. We anticipated that higher quality mother-child interactions would be associated with more optimal behavioral trajectories over time. We also hypothesized that autism status would be associated with higher levels of behavioral difficulties and slower rates of change.

\section{Methods}

\section{Participants and Procedure}

Participants were 55 mother-child dyads drawn from an ongoing longitudinal study of families of children with fragile $\mathrm{X}$ syndrome. Families were recruited from across the United States and could be included in the study if the parent was the biological mother of a child diagnosed with FXS between the ages of 1 and 4 years of age. Diagnosis of FXS was confirmed based on testing performed at the Rush University Laboratory of Dr. Elizabeth Berry-Kravis. Repeated measures data were gathered during home visits with families approximately every 18 months, resulting in 6 to 8 waves of data across childhood and into adolescence. At the most recent wave of data collection, children were between the ages of 13 and 18 years. For the present analysis, child behaviors were measured between the ages of 6 and 18 years and parenting behavior and child autism symptoms were measured during mother-child interactions when children were 2 to 6 years.

At the time of the first measurement of child behavior, children had a mean age of 7.28 years $(\mathrm{SD}=0.86)$, with a range of ages from 6.0 to 9.0. There were 19 children in 
the sample who had a diagnosis of autism. The majority of children were male $(80 \%)$ and White $(87 \%)$, with $4 \%$ of the sample being African American and 8\% being multi-racial. At the last wave of data collection, almost all children (93\%) had an individualized education program and $50 \%$ of children participated in general education settings. Almost three quarters of children were taking medications. As reported in Table 1, family pre-tax income ranged from less than $\$ 15,000$ to greater than $\$ 100,000$, with nearly 50\% earning greater than $\$ 80,000$ annually. Mothers varied in education level and marital status, as shown in Table 1.

\section{Measures}

\section{Child Behavior Problems}

Child behavior problems between the ages of 6 and 18 years were measured using subscales of the Child Behavior Checklist (CBCL; Achenbach \& Rescorla, 2001). Mothers indicated how true it was that their son/daughter exhibited behaviors in the past 6 months. Each item is rated on 3-point scale, 0 (not true), 1 (somewhat or sometimes true), 2 (very true or often true). Higher scores on each sub-scale of the CBCL indicate more severe behavior problems. Scores for the following scales were computed: attention problems, aggressive behavior, and anxious/depressed. Standard scores were calculated for descriptive purposes and raw scores were utilized in the longitudinal analyses. Scores of 64 or more are considered to be in the clinical range. Reliability and validity for the CBCL is well-established (Achenbach \& Rescorla, 2001). It is important to note that the Achenbach scales were designed to measure a variety of psychological difficulties such as anxiety and depression in the general population.

\section{Parenting}

Parenting behavior was measured during mother-child interactions when children were aged 2 to 6 years. Mothers and their children completed three structured tasks together and
Table 1 Demographic information from middle childhood data point

\begin{tabular}{|c|c|c|c|}
\hline Factor & Level & Percent $(\%)$ & $\mathrm{n}$ \\
\hline \multirow[t]{5}{*}{ Child race* } & Native American & 0 & 0 \\
\hline & Asian & 2 & 1 \\
\hline & Native Hawaiian or Pacific Islander & 2 & 1 \\
\hline & Black & 10 & 5 \\
\hline & White & 87 & 48 \\
\hline \multirow[t]{2}{*}{ Child ethnicity } & Hispanic & 4 & 2 \\
\hline & Not Hispanic & 96 & 52 \\
\hline \multirow[t]{5}{*}{ Mother race* } & Native American & 2 & 1 \\
\hline & Asian & 2 & 1 \\
\hline & Native Hawaiian or Pacific Islander & 2 & 1 \\
\hline & Black & 4 & 2 \\
\hline & White & 89 & 48 \\
\hline \multirow[t]{2}{*}{ Mother ethnicity } & Hispanic & 4 & 2 \\
\hline & Not Hispanic & 96 & 52 \\
\hline \multirow[t]{3}{*}{ Mother education in years } & 8 to 12 & 15 & 8 \\
\hline & 13 to 16 & 54 & 29 \\
\hline & 17 to $20+$ & 26 & 14 \\
\hline \multirow[t]{5}{*}{ Mother marital status } & Single & 4 & 2 \\
\hline & Married & 72 & 39 \\
\hline & Divorced & 19 & 10 \\
\hline & Separated & 2 & 1 \\
\hline & Engaged & 4 & 2 \\
\hline \multirow[t]{6}{*}{ Household income } & $<\$ 15,000$ & 6 & 3 \\
\hline & $\$ 15,000$ to $\$ 30,000$ & 13 & 7 \\
\hline & $\$ 30,000$ to $\$ 50,000$ & 13 & 7 \\
\hline & $\$ 50,000$ to $\$ 80,000$ & 19 & 10 \\
\hline & $\$ 80,000$ to $\$ 100,000$ & 15 & 8 \\
\hline & $>\$ 100,000$ & 34 & 18 \\
\hline
\end{tabular}

*Participants could indicate more than one race 
one naturalistic session. For this analysis, the naturalistic session was analyzed. All naturalistic interactions were video-recorded and coded by two trained researchers. Scores for maternal traits were assessed through a categorical coding system that evaluated maternal affect, flexibility, warmth, discipline/control, guidance, and use of punitive tone which was adapted from Landry (Landry et al., 2000). Each trait was scored along a 5-point Likert scale, with higher scores indicating more positive levels of the trait (i.e., more flexibility, more positive affect, higher warmth, lower use of punitive tone). Specific codes for maternal affect, warmth and flexibility were included in the present analysis. Each $30 \mathrm{~min}$ interaction was segmented into 10 min sections which were scored independently of one another for maternal affect, warmth, and flexibility. Scores were averaged across the 10 min segments. A reliability coder independently scored $35 \%$ of videos for all maternal traits. Intraclass correlations (ICCs) were derived to assess reliability of the scoring. The ICC for affect was 0.96; for warmth it was 0.84 ; and for flexibility it was 0.85 .

\section{Autism Status}

Autism Status $(1=$ yes, $0=$ no $)$ was determined based on administration of the Childhood Autism Rating Scale (CARS; Schopler et al., 1988). The CARS is a direct observation rating scale of autism-related behavior. The CARS was administered at each home-visit and was consensus scored by the two researchers who attended the home visit. Early CARS scores obtained over the first three observations through about age 6 years were averaged for use in this analysis. Scores of 30 or above were classified as "yes" for autism status.

\section{Analysis Plan}

First, to describe the profile of behavioral problems across childhood and adolescence, standardized scores were examined for each subscale of the CBCL (attention, anxious/depressed, aggression) at nine different age periods separated by 18 months (e.g., 6 years to 7.5 years). We also examined the percent of the sample who were experiencing clinically significant problems at each age period. Second, multi-level models were used to model the shape of the problem behavior trajectories over time using SAS PROC MIXED. The observations for each individual in the current data can be viewed as repeated measurements (Level 1) within individuals (Level 2). Age was centered at 18 years so that all intercepts are interpreted as level of problem behavior at 18 years. This age was chosen to examine problem behavior at the end of the adolescent period.

Initially an empty means, random intercept model (Model 1) was evaluated to calculate ICC. Next Model 2 evaluated the growth parameters. Fixed effects terms for intercept, linear growth, and quadratic growth were examined for all outcomes with only significant effects maintained in the model. Random effects for intercept, slope, and covariance between intercept and slope were added based on the fixed effects in the model. That is, the trajectory with age was modeled in the fixed effects while allowing individual intercepts and slopes to vary (random effects) as needed. Model 3 evaluated terms for the fixed effect of autism and maternal indicators for affect, warmth and flexibility influencing the intercept and slope when part of the model. Restricted maximum likelihood estimation was used to evaluate random effects and maximum likelihood estimation was used to evaluate fixed effects. Model comparisons were made using two types of indices: the deviance statistic, or change in the -2 log likelihood, and the Bayesian information criterion. Only new variables significantly improving the model were retained at each step of the model building. Parameter estimates, standard errors, and probability values for each of the effects are shown in Tables 3, 4.

\section{Results}

Figure 1 shows the proportion of the sample with clinicallysignificant behavior problems by age. At age 6 , almost two thirds of children were exhibiting clinically significant attention problems but by the end of adolescence, less than $20 \%$ of the sample was in the clinical range for these problems. For anxious/depressed symptoms, a third of children displayed clinically significant problems at age 12 but by the end of adolescence, less than $20 \%$ were displaying clinically-significant symptoms. Aggressive behavior was less common, with less than a third of the sample displaying problems in the clinical range during middle-childhood and less than $10 \%$ displaying such problems by the end of adolescence.

However, many youth continued to have behavioral difficulties, even though average trends reflected improvement during the study period. Further examination of clinically significant scores revealed that $33 \%$ of the sample had clinically significant attention problems on $75 \%$ or more of their occasions. $11 \%$ of the sample had clinically significant anxious/depressed problems on $75 \%$ or more of their occasions. Again, aggressive problems were less common, with only $7 \%$ of the sample displaying clinically significant aggression on $75 \%$ or more of their occasions.

Figures 2, 3, and 4 show the CBCL raw scores for Aggressive Behavior, Attention Problems, and Anxious/ Depressed over time for participating children with sex denoted by line color and style. The Attention Problem trajectories declined somewhat over time and most females tended to have fewer attention problems than most males. 
Fig. 1 Proportion of the sample with clinically-significant behavior problems on the Child Behavior Checklist by age
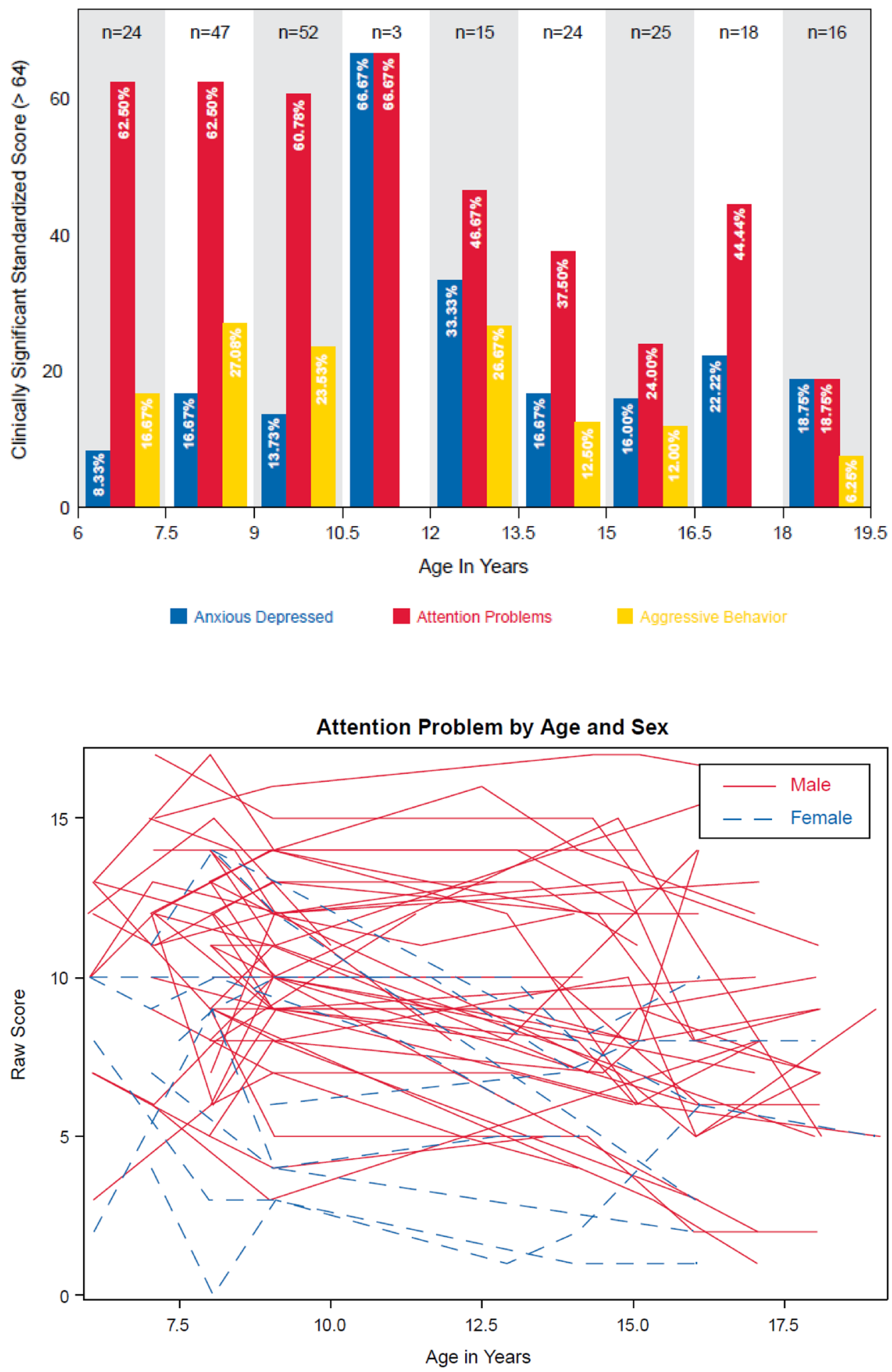

Fig. 2 Attention Problems on the Child Behavior Checklist by Age and Sex
Anxious/Depressed scores declined somewhat over the earlier ages and were somewhat stable at the older ages with some females tending to have higher scores than most males. The Aggressive Behavior trajectories were generally declining for most participants and females were dispersed throughout the distribution of aggressive behavior.

\section{Attention Problems}

The ICC for Attention Problems was 0.61, indicating that 61 percent of the variance in attention problems was between persons. As shown in Table 2, the growth model indicated that the intercept at age 18 was 7.57 and that 
Fig. 3 Aggressive Behavior on the Child Behavior Checklist by Age and Sex

Fig. 4 Anxious Depressed on the Child Behavior Checklist by Age and Sex
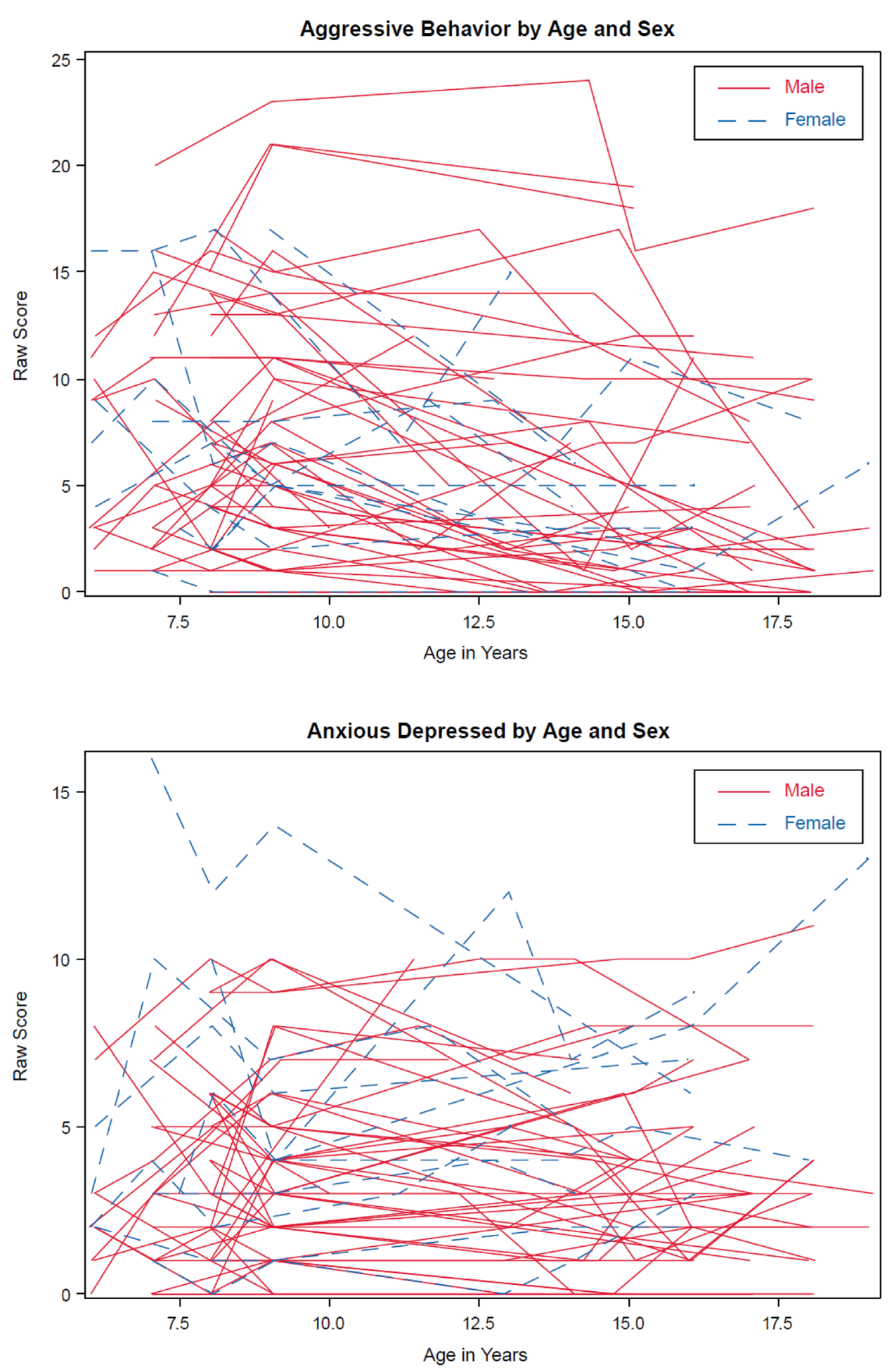

across all people, attention problems were significantly declining at age 18 . There was significant variability in intercepts, slopes, and covariance between intercepts and slopes. In Model 3, the autism group was significantly associated with intercepts such that individuals in the autism group had a predicted intercept of 9.59 and those in the No Autism group had a predicted intercept of 6.44
(3.15 lower). Maternal flexibility was related to slopes such that individuals with mothers who were higher in flexibility had greater declines in attention problem scores. The addition of autism status and flexibility reduced the variance in intercepts by $20 \%$, the covariance between slopes and intercepts by $39 \%$, and the variance in slopes 
Table 2 Fixed effect estimates (top) and variance-covariance estimates (bottom) for attention problems

\begin{tabular}{llll}
\hline & Model 1 & Model 2 & Model 3 \\
\hline Fixed effects & & & \\
Level 1 & & & \\
$\quad$ Intercept & & $7.57(.57)^{* * *}$ & $9.59(87)^{* * *}$ \\
$\quad$ Linear & & $-.24(.05)^{* * *}$ & $-.12(.08)$ \\
Predictors & & & $-3.15(1.09)^{* *}$ \\
Autism (no) on intercept & & & $-.19(.10)^{\mathrm{t}}$ \\
Autism (no) on slope & & & $-.67(.80)$ \\
Flexibility on intercept & & & $-.14(.07)^{*}$ \\
Flexibility on slope & $8.44(1.90)^{* * *}$ & $12.97(3.36)^{* * *}$ & $10.39(2.90)^{* * *}$ \\
Random effects & & $.49(.24)^{*}$ & $.30(.20)$ \\
Intercept & & $.06(.03)^{*}$ & $.04\left((02)^{*}\right.$ \\
Covariance int and slope & $3.45(.44)^{* * *}$ & $3.46(.44)^{* * *}$ \\
Slope & $5.40(.59)^{* * *}$ & & 1063.3 \\
Residual & & 1075.6 & 1103.1 \\
Model fit & 1120.8 & 1099.6 & \\
- 2 log likelihood & 1132.7 & & \\
Bayesian information criterion & & & \\
\hline
\end{tabular}

Model 1=Empty means model, Model $2=$ Growth Model, Model $3=$ Predictor Model ${ }^{\mathrm{t}} p=.051 ; * p<.05, * * p<.01, * * * p<.001$ by $33 \%$. Notably, warmth and affect were not significant in earlier models and were not retained in the final model.

Figure 5 shows predicted trajectories for attention problems over time for individuals with and without ASD who experience relatively high (1 standard deviation above the mean) vs. low (1 standard deviation below the mean) maternal flexibility. As can be seen in Fig. 5, individuals who have autism whose mothers are low in flexibility maintain high levels of attention problems. Children with autism whose mothers were highly flexible have similar rates of attention problems to children without autism whose mothers were low in flexibility.

\section{Anxious/Depressed}

The ICC for Anxious/Depressed scores was 0.65 indicating that 65 percent of the variance in anxious/depressed scores was between persons. As shown in Table 3, Model 2 indicated that anxious/depressed scores were stable at age 18 with no significant slope and an intercept of 3.91. Examination of the random effects indicated that there was
Fig. 5 Predicted Attention Problems on the Child Behavior Checklist by Flexibility and Autism Status

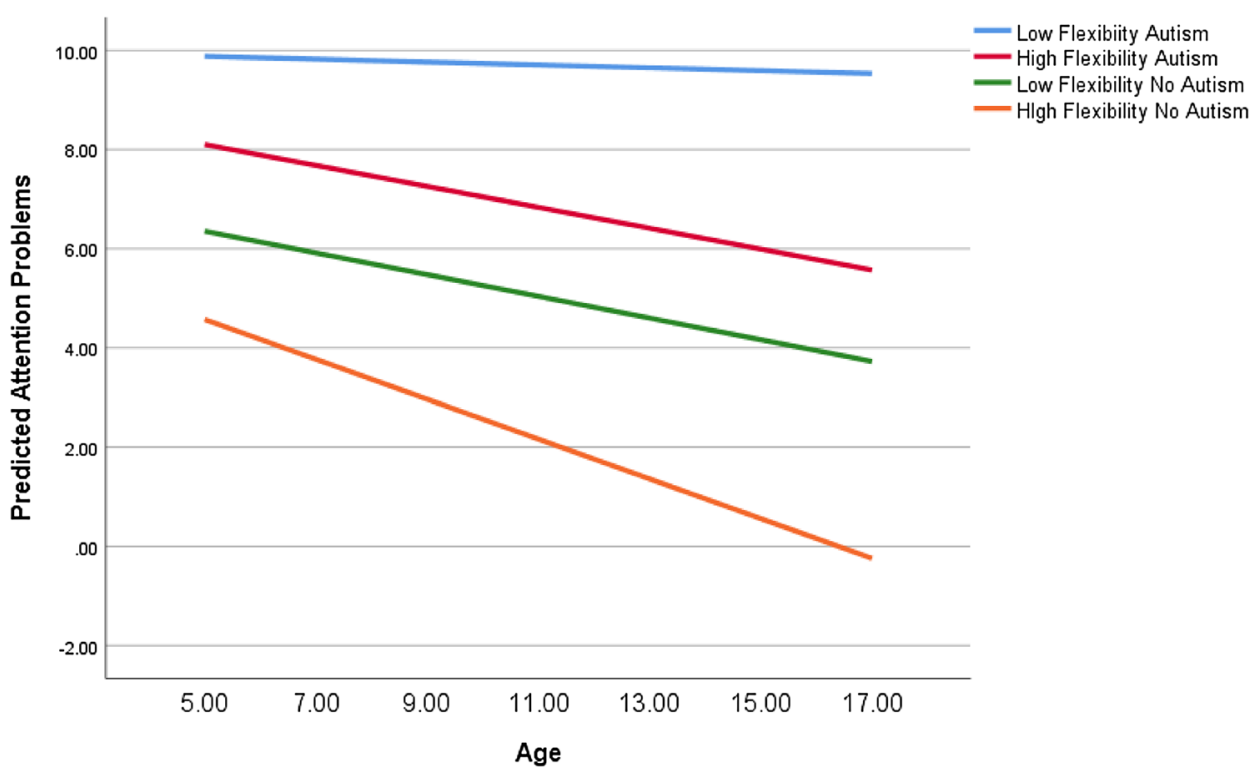


Table 3 Fixed effect estimates (top) and variance-covariance estimates (bottom) for anxious/ depressed

\begin{tabular}{llll}
\hline & Model 1 & Model 2 & Model 3 \\
\hline $\begin{array}{l}\text { Fixed effects } \\
\text { Level 1 } \\
\quad \text { Intercept }\end{array}$ & & $3.91(.36)^{* * *}$ & $3.89(.34)^{* * *}$ \\
$\quad$ Linear & & & \\
Predictors & & & $1.18(.53)^{*}$ \\
Flexibility on intercept & & & \\
Random effects & & $6.14(1.34)^{* * *}$ & $3.53(1.23)^{* * *}$ \\
$\quad$ Intercept & $6.14(1.34)^{* * *}$ & $3.33(.36)^{* * *}$ & $3.33(.36)^{* * *}$ \\
$\quad$ Residual & $3.33(.36)$ & & 1015.4 \\
Model fit & & 1020.2 & 1031.4 \\
$\quad$ 2 log likelihood & 1020.2 & 1032.1 & \\
Bayesian information criterion & 1032.1 & & \\
\hline
\end{tabular}

Model 1=Empty means model, Model 2=Growth Model, Model $3=$ Predictor Model

$* p<.05, * * p<.01, * * * p<.001$ significant variability in intercepts across individuals. In Model 3, maternal flexibility was a significant predictor of intercepts with individuals whose mothers were higher in flexibility tending to have higher anxious/depressed scores. The addition of maternal flexibility to the model reduced the variance in intercepts by $10 \%$. Once again, warmth and affect were not significant in earlier models and were not retained in the final model.

\section{Aggression}

The ICC for Aggressive Behavior was 0.70, indicating that 70 percent of the variance in aggression was between persons. As shown in Table 4, the growth model indicated that the average aggression score at age 18 was 4.64 and that aggressive behavior was significantly declining at age 18. Random effects indicated that there was significant variability in both intercepts and slopes at age 18 . We attempted to predict intercept and slope from autism status, maternal affect, maternal warmth, and maternal flexibility in Model 3. The only significant effect was for autism status on intercepts such that the predicted Aggressive Behavior score at age 18 (intercept) for the ASD group was 7.06, while for the No Autism group the intercept was 3.29. Adding the effect of autism status on intercepts to the model, reduced the variance in intercepts by $17 \%$.
Table 4 Fixed effect estimates (top) and variance-covariance estimates (bottom) for aggression

\begin{tabular}{llll}
\hline & Model 1 & Model 2 & Model 3 \\
\hline $\begin{array}{l}\text { Fixed effects } \\
\text { Level 1 }\end{array} \quad$ & & \\
$\quad$ Intercept & & $4.64(.72)^{* * *}$ & $7.06(1.07)^{* * *}$ \\
$\quad$ Linear & & $-.30(.06)^{* * *}$ & $-.31(.06)^{* * *}$ \\
Predictors & & & $-3.77(1.29)^{* *}$ \\
$\quad$ Level 2 & & & \\
$\quad$ Autism (No) on intercept & & & $16.57(3.96)^{* * *}$ \\
Random effects & $21.52(4.60)^{* * * *}$ & $20.00(4.57)^{* * * *}$ & $.06(.03)^{*}$ \\
$\quad$ Intercept & & $.07(.03)^{*}$ & $6.62(.83)^{* * *}$ \\
$\quad$ Slope & $9.22(1.00)^{* * *}$ & $6.56(.83)^{* * *}$ & \\
$\quad$ Residual & & & 1215.8 \\
Model fit & 1259.6 & 1223.8 & 1239.8 \\
$\quad$ 2 log likelihood & 1271.6 & 1243.7 & \\
Bayesian information criterion & & & \\
\hline
\end{tabular}

Model 1=Empty means model, Model $2=$ Growth Model, Model $3=$ Predictor Model

$* p<.05, * * p<.01, * * * p<.001$ 


\section{Discussion}

The present study documents important patterns of behavioral change across childhood and into adolescence for individuals with FXS. Attention problems were very high in this sample, with almost two thirds of children experiencing clinically significant problems with attention during the elementary school years. Approximately one quarter of children displayed clinically-significant aggressive behavior during the same time frame. Importantly, we found that attention problems and aggressive behavior declined steadily from childhood through adolescence, consistent with other research that has found general trends of improvements in the behavioral phenotype with age (Fielding-Gebhardt et al., 2019; Smith et al., 2016; Usher et al., 2020). In contrast, anxious/depressed behavior demonstrated relative stability over the same period. Taken together these findings highlight the value of looking at specific categories of behaviors of youth with FXS and how they may uniquely change over time.

Consistent with prior research (Smith et al., 2012a, 2012b; Usher et al., 2020), autism status played a significant role in predicting level of child behavior problems, such that individuals with co-occurring autism displayed higher levels of all three problem behavior scales than those without autism. However, although autism status was associated with greater difficulties overall, it was not a significant predictor of slope for any of the behaviors we studied. This suggests that on average both children with and without co-occurring autism may experience improvement in behavioral functioning as they move through childhood and adolescence.

We also found that maternal flexibility during mother-child interactions during early childhood was associated with greater reductions in attention problems later in childhood and adolescence. Importantly, this was particularly true for children with FXS and autism, such that children with FXS and autism whose mothers were highly flexibly had attention problems similar to children with FXS without autism whose mothers were low in flexibility. This is consistent with studies of other domains which have shown that maternal flexibility is related to improvements over time (e.g., Warren et al., 2010). In contrast, we found that mothers with higher levels of flexibility during early childhood had children with higher levels of anxious/depressed problems at age 18. This is surprising, as we anticipated that more flexibility would relate to trajectories of improvement in these challenges. Additional research is needed to understand what factors relate to change in anxious/depressed problems and possible mediating or confounding variables that impact the relationship between maternal behavior and the emergence of anxiety and depression in children with FXS. Importantly, although the number of girls in our sample was too small for conducting subgroup analyses, it is valuable to note that girls had higher anxious/depressed scores than boys. Future research should explore how gender may impact mother-child interactions and patterns of behavioral challenges over time.

In the present study, parenting (i.e., maternal affect, warmth and flexibility) was not related to aggressive behavior. It may be that endogenous factors or other environmental factors such as daily routines are more salient in the development and maintenance of aggressive behaviors. Also, it is interesting to note that maternal affect and maternal warmth were not statistically significant predictors in any of the models, either of intercept or slope. This is surprising given some prior work highlighting the role of maternal warmth, specifically, in behavioral outcomes for individuals with FXS and other intellectual and developmental disabilities (IDD; e.g., Greenberg et al., 2012; Smith, Hong, Greenberg, \& Mailick, 2016). It may be that differences in the measures of warmth used in the current study reflected different aspects of the relationship than measures used in prior work. For example, in the current study warmth was captured by coding of enthusiasm, proximity to the child, engagement, joy, pleasure derived from the child, and physical affection during an interaction. Prior studies have frequently utilized the Five Minute Speech Sample (Magaña et al., 1986) as a measure of warmth, wherein parents describe their relationship with an adolescent or adult child. Further, in our study, the parent-child interaction data were collected during early childhood, and behavioral problem trajectories were measured later (age 6 to 18). Since longitudinal data on parenting/ mother-child interactions were not included in the models in the current study, it is difficult to know how parenting behavior may have changed with time and had influence on concurrent functioning. Additional research is needed to longitudinally follow both the changes in the quality of parent-child interactions as children move from early childhood into adolescence and changes in behavioral problems.

As noted above, many children in our sample continued to display clinically significant behavioral challenges even at the end of adolescence, particularly in the areas of attention problems and anxious/depressive problems. Findings suggest that educators, physicians and other service providers should routinely monitor for co-occurring behavioral challenges across childhood and adolescence and provide diagnostic and intervention services as part of educational and treatment plans. Further, given that past work has indicated the significant stress that child behavior problems place on parents of children and youth with FXS (Abbeduto et al., 2004; Hartley et al., 2012; Hartley et al., 2012a, 2012b; Smith et al., 2012), these dyads may be particularly important to target for family support services and 
behavioral interventions. Especially relevant in a COVID-19 context, telehealth interventions may be an important vehicle for families of individuals with FXS (Nelson et al., 2018). Parent-delivered telehealth interventions have successfully reduced behavioral challenges in children with FXS (DiezJuan et al. 2014) as well as in individuals in the broader IDD population (Ramdoss et al., 2012; Kagohara et al., 2013). Understanding effective timing, dosage, and delivery of behavioral interventions through telehealth is a critical area for future research (McDuffie et al., 2016).

\section{Limitations and Conclusions}

The present study has several limitations. The sample size was relatively small, with too few females to directly test for differences in patterns of change. The sample was also primarily White, limiting generalizability. Although the temporal ordering of variables is a strength of the study (i.e., parenting measured during early childhood, behaviors measured during middle childhood and adolescence), the present study did not test the impact of parenting during adolescence or reverse models of direction of effects, that is, that behavior problems may have influenced mother-child interactions and the severity of observed autism symptoms. Additionally, child behavior problems were based on maternal report and could be strengthened by the addition of other reports or observational approaches. Juxtaposed with these limitations are several strengths. The present study included multiple waves of data spanning over a decade. Mother-child interactions and autism status were measured prior to the collection of the child behavior data using observational methods. Taken together the present study highlights both potential for improvement in specific challenging behavior for youth with FXS as well as the need for ongoing interventions to support families.

Author Contributions All authors contributed to the study conception, design, and interpretation of data. Analysis was conducted by KH and the first draft of the manuscript was written by LD. All authors commented on previous versions of the manuscript. All authors read and approved the final manuscript.

Funding This study is supported by the following NICHD Grants: P30 HD00310, P30 HD02538, and R01 HD084563 (PIs REDACTED) and by NICDC T32 DC000052 (PI: Mabel Rice).

\section{References}

Abbeduto, L., Seltzer, M. M., Shattuck, P., Krauss, M. W., Orsmond, G., \& Murphy, M. M. (2004). Psychological well-being and coping in mothers of youths with autism, Down syndrome, or fragile X syndrome. American Journal on Mental Retardation, 109(3),
237-254. https://doi.org/10.1352/0895-8017(2004)109<237: PWACIM>2.0.CO;2.

Achenbach, T. M., \& Rescorla, L. A. (2001). Manual for ASEBA School-Age Forms \& Profiles. University of Vermont, Research Center for Children, Youth, \& Families.

Bailey, D. B., Raspa, M., Olmsted, M., \& Holiday, D. B. (2008). Cooccurring conditions associated with FMR1 gene variations: Findings form a national parent survey. American Journal of Medical Genetics Part A, 146A, 2060-2069.

Brady, N., Warren, S. F., Fleming, K., Keller, J., \& Sterling, A. (2014). The effect of sustained maternal responsivity on later vocabulary development in children with Fragile X syndrome. Journal of Speech Language Hearing Research, 57(1), 212-226.

Crawford, D. C., Acuna, J. M., \& Sherman, S. L. (2001). FMR1 and the fragile $\mathrm{X}$ syndrome: Human genome epidemiology review. Genetics in Medicine, 3, 359-371.

Demark, J. L., Feldman, M. A., \& Holden, J. J. A. (2003). Behavioral relationship between autism and fragile $\mathrm{X}$ syndrome. American Journal on Mental Retardation, 108, 314-326.

Fernandez-Carvajal, I., Walichiewicz, P., Ziaosen, Z., Pan, R., Hagerman, P. J., \& Tassone, F. (2009). Screening for expanded alleles of the FMR1 gene blood spots from newborn males in a Spanish population. Journal of Molecular Diagnostics, 11, 324-329.

Fielding-Gebhardt, H., Warren, S. F., \& Brady, N. (2020). Child challenging behavior influences maternal mental health and relationship quality over time in Fragile X Syndrome. Journal of Autism and Developmental Disorders, 50(3), 779-797.

Greenberg, J. S., Seltzer, M. M., Baker, J. K., Smith, L. E., Warren, S. F., Brady, N., \& Hong, J. (2012). Family environment and behavior problems in children, adolescents, and adults with fragile $\mathrm{X}$ syndrome. American Journal on Intellectual and Developmental Disabilities, 117(4), 331-346. https://doi.org/10.1352/1944-7558117.4.331

Hagerman, P. J. (2008). The fragile X prevalence paradox. Journal of Medical Genetics, 45, 498-499.

Hardiman, R. L., \& McGill, P. (2018). How common are challenging behaviors amongst individuals with Fragile X Syndrome? A systematic review. Research in Developmental Disabilities, 76, 99-109.

Harris, S. W., Hessl, D., Goodlin-Jones, B., Ferranti, J., Bacalman, S., Barbato, I., Tassone, F., Hagerman, P. J., Herman, H., \& Hagerman, R. J. (2008). Autism profiles of males with fragile $\mathrm{X}$ syndrome. American Journal on Mental Retardation, 113(6), 427-438,

Hartley, S. L., Seltzer, M. M., Head, L., \& Abbeduto, L. (2012). Psychological well-being in fathers of adolescents and young adults with Down syndrome, fragile X syndrome, and autism. Family Relations, 61(2), 327-342. https://doi.org/10.1111/j.1741-3729. 2011.00693.x

Hartley, S. L., Seltzer, M. M., Hong, J., Greenberg, J. S., Smith, L., Almeida, D., Coe, C., \& Abbeduto, L. (2012). Cortisol response to behavior problems in FMR1 premutation mothers of adolescents and adults with fragile $\mathrm{X}$ syndrome: A diathesis-stress model. International Journal of Behavioral Development, 36(1), 53-61. https://doi.org/10.1177/0165025411406857

Hartley, S. L., Seltzer, M. M., Raspa, M., Olmsted, M. G., Bishop, E. E., \& Bailey, D. B. (2011). Exploring the adult life of men and women with fragile $\mathrm{X}$ syndrome: Results from a national survey. American Journal on Intellectual and Developmental Disabilities., 116, 16-35.

Hernandez, R. N., Feinberg, R. L., Vaurio, R., Passanante, N. M., Thompson, R. E., \& Kaufmann, W. E. (2009). Autism spectrum disorder in fragile X syndrome: A longitudinal evaluation. American Journal of Medical Genetics Part A, 149, 1125-1137.

Kagohara, D. M., van der Meer, L., Ramdoss, S., O'Reilly, M. F., Lancioni, G. E., Davis, T. N., et al. (2013). Using iPods((R)) and 
iPads $((\mathrm{R}))$ in teaching programs for individuals with developmental disabilities: A systematic review. Research in Developmental Disabilities, 34(1), 147-156.

Kaufmann, W. E., Cortell, R., Kau, A. S. M., Bukelis, I., Tierney, E., Gray, R. M., Cox, C., Capone, G. T., \& Stanard, P. (2004). Autism spectrum disorder in fragile $\mathrm{X}$ syndrome: Communicaiton, social interaction, and specific behaviors. American Journal of Medical Genetics, 129, 225-234.

Landry, et al. (2000). Early maternal and child influences on children's later independent cognitive and social functioning. Child Development, 71, 358-375.

Magaña, A. B., Goldstein, M. J., Karno, M., Miklowitz, D. J., Jenkins, J., \& Falloon, I. R. H. (1986). A brief method for assessing expressed emotion in relatives of psychiatric patients. Psychiatry Research., 17, 203-212.

McDuffie, A., Abbeduto, L., Lewis, P., Kim, J.-S., Kover, S. T., Weber, A., \& Brown, W. T. (2010). Autism spectrum disorder in children and adolescents with fragile $\mathrm{x}$ syndrome: Within-syndrome differences and age-related changes. American Journal on Intellectual and Developmental Disabilities, 115, 307-327.

Nelson, S., McDuffie, A., Banasik, A., Feigles, R. T., Thurman, A. J., \& Abbeduto, L. (2018). Inferential language use by school-aged boys with fragile $\mathrm{X}$ syndrome: Effects of a parent-implemented spoken language intervention. Journal of Communication Disorders, 72, 64-76.

Ramdoss, S., Machalicek, W., Rispoli, M., Mulloy, A., Lang, R., \& O'Reilly, M. (2012). Computer-based interventions to improve social and emotional skills in individuals with autism spectrum disorders: A systematic review. Developmental Neurorehabilitation, 15(2), 119-135.

Schopler, E., Reichler, R., Tochen Reener, B. (1988). The childhood autism rating scale. Western Psychological Services.

Smith, L. E., Barker, E. T., Seltzer, M. M., Abbeduto, L., \& Greenberg, J. S. (2012). Behavioral phenotype of fragile X syndrome in adolescence and adulthood. American Journal on Intellectual and Developmental Disabilities, 117(1), 1-17. https://doi.org/10. 1352/1944-7558-117.1.1
Smith, L. E., Seltzer, M. M., \& Greenberg, J. S. (2012). Daily health symptoms of mothers of adolescents and adults with fragile $\mathrm{X}$ syndrome and mothers of adolescents and adults with autism spectrum disorder. Journal of Autism and Developmental Disorders, 42(9), 1836-1846. https://doi.org/10.1007/s10803-011-1422-7

Smith, L. E., Hong, J., Greenberg, J. S., \& Mailick, M. R. (2016). Change in the behavioral phenotype of adolescents and adults with FXS: Role of the family environment. Journal of Autism and Developmental Disorders, 46(5), 1824-1833. https://doi.org/10. 1007/s10803-016-2714-8

Sterling, A., Warren, S. F., Brady, N., \& Fleming, K. (2013). Influences on maternal responsivity in mothers of children with Fragile $\mathrm{X}$ Syndrome. American Journal on Intellectual and Developmental Disabilities, 118(4), 310-326.

Usher, L. V., DaWalt, L. S., Hong, J., Greenberg, J. S., \& Mailick, M. R. (2020). Trajectories of change in the behavioral and health phenotype of adolescents and adults with fragile $\mathrm{X}$ syndrome and intellectual disability: Longitudinal trends over a decade. Journal of Autism and Developmental Disorders, 50(8), 2779-2792.

Warren, S. F., Brady, N., Sterling, A., Fleming, K., \& Marquis, J. (2010). Maternal responsivity predicts language development in young children with fragile X syndrome. American Journal of Intellectual and Developmental Disabilities, 115(1), 54-75.

Warren, S. F., Brady, N., Fleming, K., \& Hahn, L. J. (2017). The longitudinal effects of parenting on adaptive behavior in children with Fragile X Syndrome. Journal of Autism and Developmental Disorders, 47(3), 768-784.

Warren, S. F., \& Brady, N. (2007). The role of maternal responsivity in the development of children with intellectual disabilities. Mental Retardation and Developmental Disabilities Research Reviews, 13(4), 330-338.

Publisher's Note Springer Nature remains neutral with regard to jurisdictional claims in published maps and institutional affiliations. 